\title{
Lensed density perturbations in braneworlds: Towards an alternative to perturbations from inflation
}

\author{
Daniel J. H. Chung \\ Michigan Center for Theoretical Physics, Ann Arbor, Michigan 48109 \\ and CERN Theory Division, CH-1211 Geneva 23, Switzerland \\ Katherine Freese \\ Michigan Center for Theoretical Physics, Ann Arbor, Michigan 48109 \\ and ISCAP, Columbia Astrophysics Laboratory, 550 West 120th Street, New York, New York 10027
}

(Received 6 March 2002; published 19 May 2003)

\begin{abstract}
We consider a scenario in which our observable universe is a 3-dimensional surface (3-brane) living in extra dimensions with a warped geometry. We explore the interesting possibility that "lensed" density perturbations from other branes may serve as possible seeds for structure formation on our observable brane (without inflation), and, in addition, provide constraints on braneworld scenarios with warped bulk geometry. Because of the warped bulk metric, any perturbation generated on one brane (or in the bulk matter) appears to an observer on a second brane to have a significantly different amplitude. We analyze lensed perturbations in the Randall-Sundrum type scenarios and the "shortcut metric" scenarios. For Lorentz violating metrics in the bulk, we find the attractive possibility that large density fluctuations that are causally produced elsewhere can lead to small density fluctuations on our brane on superhorizon (acausal) length scales, as required by structure formation. Our most interesting result is that the "shortcut metrics" in which geodesics traverse the extra dimensions has the chance of providing an alternative to inflation with two important features: a possible solution to the horizon problem and a possible mechanism to generate large scale perturbations necessary for structure formation.
\end{abstract}

DOI: 10.1103/PhysRevD.67.103505

\section{INTRODUCTION}

In the early universe, fluctuations in the matter density are thought to grow via gravitational instability to give rise to the formation of galaxies and other large scale structure. Cosmic microwave background measurements [1] constrain the amplitude of the initial density perturbations to be

$$
\delta \rho / \rho \sim 10^{-5}
$$

In order to explain structure, these fluctuations must have existed on large "superhorizon" length scales, larger than causally connected (in the naive sense) regions in the early universe. One mechanism to generate such fluctuations is inflationary cosmology [2], in which a small causally connected region grow superluminally at a very early time to encompass regions larger than our observable universe today. Inflation is able to solve the horizon, flatness, and monopole problems. However, in most inflationary models, the smallness of the perturbations in Eq. (1) is difficult to maneuver. The magnitude of the perturbations is proportional to the ratio of the height of the potential to the (width of the potential $)^{4}$ [3]. Since the height and width are usually given by the same mass scale, the smallness of this parameter is hard to explain. Natural inflation [4] is a model in which a pseudoNambu-Goldstone boson provides an explanation for the two different required mass scales. However, in generic models, the small number in Eq. (1) is problematic.

One of the main points in this paper is to describe a different generation mechanism for density fluctuations which we will call "lensed perturbations." We consider a scenario in which our observable universe is a 3-dimensional surface
PACS number(s): 95.35.+d, 14.80.Ly, 98.70.Sa

(3-brane) living in higher dimensions. In particular, we focus on a warped geometry for the extra dimensions. We work in 5 dimensions, but the idea may be generalizable to higher dimensions.

Perhaps the most interesting application of lensed perturbations is to "shortcut metrics" with geodesics that traverse the extra dimensions [7]. If lensed density perturbations can be successfully embedded, such metrics have a chance of providing an alternative to inflation with respect to two interesting features: a possible solution to the horizon problem and generation of density fluctuations that can give rise to structure formation. As we will explain, although we do not realize the full density fluctuation scenario in this paper, this paper serves as a step in this direction.

For concreteness, we consider a metric of the form

$$
d s^{2}=-n^{2}(u, t) d t^{2}+a^{2}(u, t) \delta_{i j} d x^{i} d x^{j}+d u^{2},
$$

where the coordinate $u$ parametrizes the extra dimension. Here, $a(u, t)$ is the scale factor of the 3-dimensional slices parallel to our brane. For most of the paper, we will treat the time dependence of the metric, at most, as a perturbation. We will take the hypersurfaces defined by $u=0$ and $u=L$ to be the world volumes of our observable universe brane and of a "hidden sector" brane. ${ }^{1}$

\footnotetext{
${ }^{1}$ By "hidden sector," we mean that the two branes do not communicate through any of the standard model fields (we here use the language in which the graviton is not considered a standard model field).
} 
The key here is to have a warped metric factor $a(u, t)$ that changes rapidly as one leaves the brane along the $u$ direction, leading to a change in comoving volume factor. An example is

$$
a(u, t)=e^{-b u}
$$

for $0 \leqslant u \leqslant L$. This has been considered previously in many different physical contexts, e.g., by Randall-Sundrum [5] and [6] in the context of the hierarchy problem and gravity localization, and by Ref. [7] in the context of the horizon problem. Consider a scenario in which our observable brane is at $u=0$ and there is a second (hidden) brane at $u=L$. With such a metric, the 3 -volume at $u=0$ is larger by a factor $e^{3 b L}$ than the volume at $u=L$ for a fixed coordinate volume; this change in comoving 3-volume is key to the lensing effect. Now, imagine that density fluctuations on very small scales but of very large amplitude (e.g. $\delta \rho_{h} /\left.\rho_{h}\right|_{L} \sim 1$ ) are produced on this hidden brane at $u=L$. Here the subscript $h$ denotes the hidden sector brane quantities. For example, these perturbations might be produced at some analog of a quark-hadron transition, which has has a horizon size of a few kilometers and hence appears useless to generating large scale density fluctuations. However, if such perturbations are generated and localized on the hidden brane, they will appear to us to be on much larger scales and of much smaller amplitude. For very long wavelength modes, the amplitude is smaller by roughly the ratio of brane volumes:

$$
\underline{\delta \rho_{h}} \sim e^{-c b L} \delta \rho_{h},
$$

where the underline refers to a projection onto our brane at $u=0$ (i.e. what the observers on the brane at $u=0$ gravitationally perceive) ${ }^{2}$ Here the factor of $c$ in the exponent is a number of order 1 determined by the ratio of volumes and the time measure which fixes the energy measurement. In addition, in the case of Lorentz violating metrics (such as used in [7]), perturbations produced on causal length scales are blown up to much larger, acausal length scales on our brane. Hence our observable universe sees large scale, small amplitude fluctuations such as are required for structure formation.

Before going on, we would like to here interject and state our notation: Throughout the paper, underlined quantities are those that have been projected elsewhere, usually from the hidden brane onto the observable brane.

In a previous paper [7], we proposed a non-inflationary solution to the cosmological horizon problem via shortcuts through the extra dimensions. A signal traveling along an extra-dimensional null geodesic may leave our 3-brane, travel into the extra dimensions, and subsequently return to a different place on our 3-brane in a shorter time than the time a signal confined to our 3-brane would take. Works related to this idea include [8-28]. One of the metrics we considered

\footnotetext{
${ }^{2}$ As usual, we do not define the brane tension to be part of these densities. Furthermore, as we will explain later, for the RandallSundrum case this enhancement cannot be measured by a low energy observer, hence can be viewed as spurious.
}

had the warped form described in Eq. (3). Hence, in such a shortcut scenario, it is possible that small scale, large amplitude perturbations generated on a hidden brane provide the necessary superhorizon small amplitude fluctuations on our brane. Any alternative to inflation should address not only the horizon problem but also perturbation generation as well as the flatness problem. In fact, it is this question that initiated the current investigation. Here we propose and analyze the features of a possible generation mechanism for the fluctuations in the shortcut scenario.

We also wish to point out that lensed perturbations may be useful even if our brane does have an inflationary period to solve the horizon and the flatness problem. If inflation cannot produce perturbations required by Eq. (1) (e.g., the height of the potential is too low), lensed perturbations projected onto our brane from elsewhere may be essential to the formation of structure in the universe.

Our results apply equally to a single brane scenario. For example, density fluctuations produced in the bulk will have the same projection on our brane (as from a hidden brane) and may thus be interesting for structure formation.

At the outset, we analyze lensed density perturbations in the RS (Randall-Sundrum) scenario for warm up since the answer is obvious and easy to interpret there. For perturbations of a given amplitude on a hidden brane with a RandallSundrum metric, we have found the size of the perturbations seen by an observer on our brane and present the results in Eqs. (55) and (56). In the first paper with warped geometry, RSI [5], the hidden brane is at $u=0$ and the observable brane is at $u=L$; i.e., the locations of the hidden and observable branes are exchanged from our previous discussion, such that the warping goes in the opposite direction and the volume is smaller on our brane. In this situation, we find that homogeneous (long wavelength) perturbations appear larger on our brane than where they are generated. However, from a low energy observer point of view, this projected larger effective density is cloaked in the warp factor suppressed volume factor such that the observed density is the same as that seen on the brane where the density locally resides. Hence, the bound coming from today's cosmological observations are same as that for the usual dark matter.

Note that one might expect an exact reversal of the lensing if the observer were in the bulk instead of on a brane at $u=0$ : if large density perturbations produced elsewhere look small on our brane, then one might expect small perturbations produced on our brane to look large as one moves further and further away from our brane. This situation applies to the second paper by Randall and Sundrum, RSII [6]. Indeed, for large wavelength modes, this reversal of lensing is true. In that case, at a far enough distance, one might fear that there are large back reaction corrections (for related work, see [29-33]). However, as was shown in Ref. [29] and as is reviewed in the Appendix, the higher momentum modes can still be suppressed with the result that there is not necessarily a large back reaction. The degree to which there is a back reaction depends upon the degree of spatial localization of the perturbation. Roughly speaking, more extended sources lead to a greater back reaction. 
In the Discussion section we briefly address the flatness problem. Although it seemed naively plausible that the difference in effective mass density between lensed and intrinsic perturbations could be used to ameliorate the flatness problem, we find that it does not help at all. We also mention a possible role of lensed perturbations in the dark energy problem. We speculate that large energy densities with a negative equation of state on the hidden sector brane (or in the bulk) may be projected to a small energy density on our brane and hence provide an explanation of the current acceleration of the universe.

Finally, we would like to emphasize that although our calculations in this paper bring out certain qualitative features of density perturbations in braneworlds, details of their implications will require more model dependent investigations by perturbing about fully time dependent backgrounds. Some work in braneworld density perturbations in time dependent backgrounds can be found in [34-40] and references therein. In this paper, for the most part, we do not consider the time evolution of the perturbations.

In Ref. [41], some generic constraints were given regarding large scale perturbations based on local Einstein equations on the brane and assumptions about the separation of the brane-localized and bulk energy components (independent stress-energy conservation equations). In particular, the curvature perturbations on "acausal" scales have been shown to be negligible. However, this conclusion requires the assumption about conservation of the brane energy momentum separately from the bulk energy-momentum [implicit in Eq. (3.17) of that reference]. This is a stringent assumption, which need not apply in general to the lensed perturbation scenario. Indeed, it is well known that freezing of the density perturbation amplitude on large scales is more tied with conservation equations rather than details of the gravitational equations.

Reference [41] does point out a generic property that the bulk energy densities have components that look like anisotropic stresses on our brane. This applies equally well to the lensed density perturbations. Furthermore, since by definition, the photons on our brane are not producing the lensed perturbations, the lensed density perturbations will behave as isocurvature perturbations. Since it is difficult to reproduce the cosmic microwave background (CMB) measurements using only isocurvature perturbations, it is not clear whether or not these perturbations will be by themselves enough to explain the observed CMB measurements. On the other hand, since the effective 4D (four dimensional) gravity must undergo significant model dependent time evolution until the time of nucleosynthesis (which obviously is before CMB decoupling), one cannot make any model independent conclusions. (Indeed, by the time of nucleosynthesis, the effective gravity may revert adiabatically to $4 \mathrm{D}$ Einstein gravity once the $S O(1,3)$ isometry violating source terms dissipate.) As stated before, we will leave time dependence analysis to a future work.

Before we outline the rest of the paper, we would like to further emphasize that because our calculations are restricted to time independent limit, the calculations are valid only far within the 5D horizon length scales (five dimensional hori- zon coming from expansion). As we further explain more carefully in the text later, in the case of Randall-Sundrum like scenarios, this means that our calculations cannot say anything about how gravitational perturbations look on super-4D-cosmological-horizon length scales (nor can any be projected from causal length scales to superhorizon scales without inflation). However, the calculations well describe how gravity looks on subhorizon scales, just as to describe planetary motion around the Sun, we need not worry about the cosmological expansion rate in a $4 \mathrm{D}$ universe.

In the shortcut scenario, the situation is different in that the spacetime region far within 5D horizon scale can correspond to a region of super-4D-cosmological-horizon (as defined on the brane) scale. Hence, just as in planetary science, far within the 5D horizon scale (due to cosmological expansion), we can to leading approximation neglect the effects of $5 \mathrm{D}$ horizon. We are in no way violating the claim that our results (given the approximation of nearly time independence) are only valid far within the 5D horizon length scales. The utility of our result to a more realistic complete model of asymmetric warping is that if the distance between the two branes in the shortcut scenario is not changing appreciably during the cosmological expansion and if the asymmetric warping is severe enough, our results will be what one should expect from a full, time-dependent analysis far within the 5D expansion horizon but still far outside of the 4D expansion horizon. (After all, the signals are only traveling less than a millimeter.)

In the next section, we begin by discussing lensed perturbations in a Randall-Sundrum metric in Sec. II. The main aim of that section is to establish notation and build intuition for the analysis of the shortcut metric perturbations. For perturbations of a given amplitude on a hidden brane with a Randall-Sundrum metric, we have found the size of the perturbations seen by an observer on our brane and present the results in Eqs. (55) and (56). Next we proceed to lensed perturbations in a shortcut metric in Sec. III. In Sec. IV, we discuss possible generation mechanisms of perturbations on the hidden brane, comment on the flatness problem, and close with a speculation of the use of lensing on the dark energy problem.

\section{DENSITY PERTURBATIONS IN THE RANDALL-SUNDRUM METRIC}

In this section, we analyze the perturbations about the Randall-Sundrum metric both from a 4D effective theory point of view and the 5D point of view. Our main objective is to establish notation and build intuition for the analysis in the next section regarding the perturbations about the shortcut metric. In particular, although a 4D effective theory description is sufficient to understand the qualitative features of the perturbations in the Randall-Sundrum scenario, since a covariant $4 \mathrm{D}$ effective theory description does not exist in the shortcut scenario, we carry out both a 4D and a 5D analysis in the Randall-Sundrum scenario as a warm up exercise to compare to the $5 \mathrm{D}$ analysis of the shortcut scenario in the next section.

We restrict our attention to 5 dimensions with the coordi- 
nate $u$ parametrizing the extra dimension. In addition, for simplicity, let the extra dimension be compactified on an $S^{1} / Z_{2}$. Consider 2 branes at orbifold fixed points, with brane 1 at $u=L_{1}$ and brane 2 at $u=L_{2}$. We would like to ask the question: given that there is a density perturbation $\delta \rho_{2}$ on brane 2, what is the "effective density perturbation" seen by the observer on brane 1 ?

We consider the perturbation about the AdS metric:

$$
d s^{2}=e^{-2 b u}\left[\eta_{\mu \nu}+h_{\mu \nu}(x, u)\right] d x^{\mu} d x^{\nu}+d u^{2} .
$$

Equation (2) reduces to this case when $a(u)=n(u)=e^{-2 b u}$ and for linearized gravity, $g_{\mu \nu}=\eta_{\mu \nu}+h_{\mu \nu}$, where $\eta_{\mu \nu}$ is the metric of Minkowski space. Here, Greek indices are four dimensional and range from $\mu=0,1,2,3$. Then any matter on the branes is treated as a perturbation to this background and serves as a source for linearised gravity.

A treatment of linearized gravity in the Randall-Sundrum background has been worked out by [29]. Taking ${ }^{3}$

$$
h_{\mu \nu}=\bar{h}_{\mu \nu}-\frac{\bar{h}}{d-2} \eta_{\mu \nu}
$$

as the definition of $\bar{h}_{\mu \nu}$,

$$
T_{\mu \nu}=S_{\mu \nu}(x) \delta\left(u-L_{2}\right)
$$

as the 5D stress tensor where $S_{\mu \nu}$ is the 4-dimensional stress tensor confined to the brane 2 at $u=L_{2}$, and

$$
T_{\mu u} \equiv 0\left(\text { e.g. }, T_{05} \equiv 0\right),
$$

they obtained

$$
\begin{aligned}
\frac{1}{\sqrt{g}} \partial_{A}\left[\sqrt{g} g^{A B} \partial_{B}\right] \bar{h}_{\mu \nu}(x, u)= & \frac{-e^{2 b u}}{M^{3}}\left[S_{\mu \nu}-\frac{1}{6} \eta_{\mu \nu} S_{\lambda}^{\lambda}\right] \\
& \times \delta\left(u-L_{2}\right)-\frac{1}{M^{3}} Q_{\mu \nu}
\end{aligned}
$$

where $Q_{\mu \nu}$ contains the delocalized (not localized by a delta function) stress energy related to the stabilization of the extra dimension, $M$ is the 5-dimensional Planck mass, and all Greek indices are raised and lowered by $\eta_{\mu \nu}$.

Let us pause here to explicitly display the warp factor that arises in the case that the source $S_{\mu \nu}$ is a perfect fluid. The five dimensional stress tensor for a perfect fluid confined to the brane located at $u=L_{2}$ can be written as

$$
T_{B}^{A}=\left[p_{2}\left(\delta_{B}^{A}-\delta^{A 5} \delta_{B 5}\right)-\left(p_{2}+\rho_{2}\right) \delta^{A 0} \delta_{B 0}\right] \delta\left(u-L_{2}\right)
$$

in the rest frame of the fluid, where $\rho_{2}$ and $p_{2}$ are energy density and pressure, respectively; i.e., $T_{B}^{A}$ $=\operatorname{diag}(-\rho, p, p, p, 0)$. Here Latin indices are 5-dimensional

\footnotetext{
${ }^{3} \mathrm{We}$ remind the reader that underlined quantities indicate projected quantities from one brane to another, and are not to be confused with the overbar here.
}

and range from $A=0,1,2,3,4$. These 5-dimensional indices are raised and lowered with the 5-dimensional metric $g_{A B}$ $=\operatorname{diag}\left(e^{-2 b u} g_{\mu \nu}, 1\right)$. Now, stress tensor $T_{\mu \nu}$ on brane 2 is the four-dimensional part of $T_{A B}=g_{A C} T_{B}^{C}$, i.e.,

$$
\begin{aligned}
T_{\mu \nu} & \equiv e^{-2 b u} \eta_{\mu A} T_{\nu}^{A}=\eta_{\mu \alpha} T_{\nu}^{\alpha} \\
& =e^{-2 b u}\left[p_{2} \eta_{\mu \nu}-\left(p_{2}+\rho_{2}\right) \eta_{0 \mu} \delta_{\nu 0}\right] \delta\left(u-L_{2}\right) \\
& =S_{\mu \nu} \delta\left(u-L_{2}\right)
\end{aligned}
$$

Hence, note that $S_{\mu \nu}$ has an additional warp factor compared to what one would naively write down.

\section{A. 4D effective description}

Here we obtain a preview of part of our results for the Randall-Sundrum case study by looking at the problem from a four-dimensional point of view. We integrate over the fifth dimension $u$ to obtain a 4D zero mode effective theory point of view. In this way one finds a result which holds in the long wavelength limit of the more general study we perform below. It is important to note that from a $4 \mathrm{D}$ zero mode effective theory point of view, $p_{2}$ and $\rho_{2}$ in Eq. (10) cannot be measured. Although this $4 \mathrm{D}$ effective theory treatment is sufficient to accurately describe the processes in the RSII case (as our fully 5D investigation will confirm), since a 4D effective theory is not a good description of gravity for the shortcut scenario, to be able to compare the shortcut scenario with the Randall-Sundrum scenario, a 5D analysis of the Randall-Sundrum scenario is required and will be carried out in the subsequent subsection.

Consider the action

$$
\begin{aligned}
S= & M^{3} \int d^{5} x \sqrt{g} R\left[g_{A B}\right]+\int d^{5} x \sqrt{g} \mathcal{L}\left[g_{A B}, \phi_{i}\right] \\
& \times \delta(u-L)+\cdots
\end{aligned}
$$

where $R$ is the Ricci scalar and $\mathcal{L}$ is the 4D matter Lagrangian. With the usual zero mode expansion ansatz $d s^{2}$ $=e^{-2 b u} \widetilde{g}_{\mu \nu}(x) d x^{\mu} d x^{\nu}+d u^{2}$, where we consider only the metric $\tilde{g}_{\mu \nu}(x)$ with no $u$-dependence, one finds

$$
S \approx \frac{M^{3}}{2 b} \int d^{4} x \sqrt{\tilde{g}} R_{4 D}\left[\tilde{g}_{\mu \nu}\right]+S_{M}+\cdots
$$

where

$$
S_{M}=\int d^{4} x \sqrt{\tilde{g}} e^{-4 b L} \mathcal{L}\left[e^{-2 b L} \tilde{g}_{\mu \nu}, \phi_{i}\right]
$$

This equation describes the 4D effective gravity interaction if the radion is stabilized. In this case, the measurable stress tensor is the variation with respect to $\delta \tilde{g}_{\mu \nu}$. Take the functional derivative with respect to $\tilde{g}_{\mu \nu}$, 


$$
\frac{\delta S}{\delta \widetilde{g}^{\mu \nu}}=\frac{M^{3}}{2 b} \sqrt{\tilde{g}}\left[R_{4 D \mu \nu}-\frac{1}{2} \widetilde{g}_{\mu \nu} R_{4 D}\right]+\frac{\delta S_{M}}{\delta \widetilde{g}^{\mu \nu}} .
$$

Hence, we arrive at the 0-0 component of the Einstein's equation

$$
R_{4 D 0}^{0}-\frac{1}{2} R_{4 D}=\frac{1}{M_{p l}^{2}} \frac{2}{\sqrt{\tilde{g}}} \frac{\delta S_{M}}{\delta \widetilde{g}^{\alpha 0}} \tilde{g}^{0 \alpha}=-\frac{\bar{\rho}^{(4 D)}}{M_{p l}^{2}}
$$

where the second equality derives from the definition of $4 \mathrm{D}$ stress tensor $\bar{\rho}_{2}^{(4 D)}$ that goes with the Einstein equations.

Now, let us vary the 5D metric to find an expression of $\rho_{2}$ as a functional of $S_{M}$ :

$$
\begin{aligned}
T_{0}^{0}=-\rho_{2} \delta(u-L) & =\frac{2}{\sqrt{g}} \frac{\delta S_{M}}{\delta g^{A 0}} g^{0 A} \delta(u-L) \\
& =\frac{2}{\sqrt{\tilde{g}} e^{-4 b L}} \frac{\delta S_{M}}{\delta \tilde{g}^{A 0}} \widetilde{g}^{0 A} \delta(u-L) .
\end{aligned}
$$

Comparing this with Eq. (18), we see

$$
\bar{\rho}^{(4 D)}=e^{-4 b L} \rho_{2} .
$$

Hence the low energy quantity $\bar{\rho}_{2}^{(4 D)}$ measured by a four dimensional observer on brane 1 at $u=0$ due to energy momentum on brane 2 at $u=L$ is suppressed.

Thus, without a detailed calculation, we already see what the answer to our question is in the zero momentum (long wavelength) limit. Since the effective action for the matter confined to the brane at $u=0$ is

$$
\int d^{4} x \sqrt{\tilde{g}} \mathcal{L}\left(\tilde{g}_{\mu \nu}, \phi_{i}\right)
$$

which is unsuppressed, we see that the energy density projected onto brane 1 from brane 2 will be suppressed by $e^{-4 b L}$. Note that this was already emphasized by Ref. [42].

Note that there is no suppression in the other direction. If there is a source at $u=0$, then the associated $\delta(u=0)$ gives rise to a factor $e^{0}=1$ so that there is no warp factor; hence a source at $u=0$ appears unsuppressed to a source at $u=L$ i.e.,

$$
\bar{\rho}^{(4 D)}=\rho(u=0) .
$$

In the following section, we perform a more complete general analysis in which we study solutions to the equations of motion. Given a source on one brane, we find the effective density on another brane that would be needed to reproduce the gravitational behavior. Below both the source and effective density are 5-dimensional objects, in that they are defined in a 5-dimensional stress tensor. We will find that a source at location $L$ appears suppressed at $u=0$, while a source at location $u=0$ appears enhanced at $u=L$. However, as discussed in the previous paragraphs, these 5-dimensional objects are not measurable observables. One must still construct the 4-dimensional object $\bar{\rho}_{2}^{(4 D)}$ which appears in the
4D effective action. Then we confirm that in the long wavelength limit one obtains a suppressed effective 4D density for a source at $u=L$ at $u=0$; we also confirm that there is no suppression or enhancement in the long wavelength limit in the observable density for a source at $u=0$ on a brane at $u$ $=L$.

\section{B. General analysis}

Now we turn to a more general study in which we study solutions to the equations of motion for density perturbations. Rather than the coarse-graining approach of the previous paragraphs, we perform a careful analysis of linearized gravity (which includes Kaluza-Klein modes). Here the fourdimensional stress tensor $S_{\mu \nu}$ includes all the matter on brane 2, and is treated as a perturbation about the much larger brane tension in the AdS background. Hence, $S_{\mu \nu}$ includes not only the usual energy density perturbation in cosmology (i.e. $\delta \rho$ in $\delta \rho / \rho$ ) but also the energy density $\rho$ as well.

We keep terms only up to linear order in the expansion in $S_{\mu \nu} / B$, where $B$ is brane tension. Since we include both $\rho$ and $\delta \rho$ in $S_{\mu \nu}$, this first order treatment restricts the validity of the analysis to the case where $\delta \rho / B>(\rho / B)^{2}$, i.e.

$$
\frac{\rho}{b^{2} M_{p l}^{2}}<\frac{\delta \rho}{\rho}
$$

where brane tension $\sim b^{2} M_{p l}^{2}$. In the rest of this paper, we will almost always refer to $S_{\mu \nu}$ as a density perturbation and will not distinguish between $\rho$ background and $\delta \rho$ unless required.

In addition, the slice expansion in 3-spatial dimensions with local (in $u$ ) expansion rate $\dot{a} / a(u)$ is encoded into $h_{i i}$ $\sim 2 \dot{a} / a\left(u, t_{\text {initial }}\right)\left(t-t_{\text {initial }}\right)+\cdots$, and the equations are only valid for time length scales smaller than $\max [a / \dot{a}]$. Hence, any conclusions that one can draw from our analysis regarding cosmological horizon physics is only qualitative.

Now we can begin to address the question: given that there is a density perturbation $\delta \rho_{2}$ on brane 2 , what is the "effective density perturbation" seen by the observer on brane 1 ? For generality, we take brane 1 to be located at $u$ $=L_{1}$ and brane 2 to be located at $L_{2}$. We define a 4 dimensional effective $S_{\mu \nu}$ as the 4-dimensional density perturbation on brane $1{ }^{-}$which reproduces the gravitational effects generated by a density perturbation $S_{\mu \nu}$ on brane 2. Explicitly, we define it as the source at $u=L_{1}$ such that

$$
\begin{aligned}
& \frac{1}{\sqrt{g}} \partial_{\alpha}\left[\sqrt{g} g^{\alpha \beta} \partial_{\beta}\right] \bar{h}_{1, \mu \nu}(x, u) \\
& =\frac{-e^{2 b u}}{M^{3}}\left[S_{-\mu \nu}-\frac{1}{6} \eta_{\mu \nu} S_{-}^{\lambda}\right] \delta\left(u-L_{1}\right)-\frac{1}{M^{3}} Q_{\mu \nu}
\end{aligned}
$$

where $\bar{h}_{1, \mu \nu}$ matches $\bar{h}_{\mu \nu}$ of Eq. (9) at $u=0$. Our aim will be to solve for $S_{\mu \nu}$. Note that although this projected stress 
tensor is fictitious, it captures the gravitational information about what the observer on brane 1 sees due to the stress tensor perturbation on brane 2. Also note that although this stress tensor is intrinsically 4 dimensional since it is the response to the variation of the induced metric, the induced metric describes the geometry of a slice of a 5 dimensional space. Hence, in that sense, this stress energy tensor is really associated with 5 dimensional physics.

First look for the Green's function satisfying

$$
\frac{1}{\sqrt{g}} \partial_{A}\left[\sqrt{g} g^{A B} \partial_{B}\right] G\left(x, u ; x_{o}, u_{o}\right)=\delta^{(4)}\left(x-x_{0}\right) \delta\left(u-u_{o}\right)
$$

or explicitly

$$
e^{2 b u}\left[\partial_{t}^{2} G-\partial_{i}^{2} G\right]+4 b \partial_{u} G-\partial_{u}^{2} G=\delta^{(4)}\left(x-x_{0}\right) \delta\left(u-u_{o}\right) .
$$

Writing $G$ as

$$
G=\int \frac{d^{4} p}{(2 \pi)^{4}} e^{i p \cdot\left(x-x_{0}\right)} H\left(p, u, u_{0}\right)
$$

(where the dot product is only over the $0-3$ coordinates $x$ and not over the $u$ coordinate), we find

$$
H^{\prime \prime}-4 b H^{\prime}+e^{2 b u}\left(p_{0}^{2}-\vec{p}^{2}\right) H=-\delta\left(u-u_{0}\right),
$$

where the prime denotes the derivative with respect to $u$. The integral of this equation yields the discontinuity in $H^{\prime}$ at $u$ $=u_{0}$, i.e., the jump condition at $u=u_{0}$. Let us denote $H_{>}$to be the solution satisfying the homogeneous equation for $u$ $>u_{0}$ and $H_{<}$to be the solution for $u<u_{0}$. Note that in the case that $u_{0}$ approaches $L_{1}$, it approaches $L_{1}$ from the right. Similarly, when $u_{0}$ approaches $L_{2}$, it approaches it from the left. Hence, we can write the boundary conditions from the jump equation as

$$
\left.\left(H_{>}^{\prime}-H_{<}^{\prime}\right)\right|_{u_{0}}=-1
$$

and

$$
H_{>}\left(u_{0}\right)=H_{<}\left(u_{0}\right) .
$$

In the presence of branes and neglecting back reaction effects due to sources on the branes, we have the additional boundary conditions at the branes on orbifold fixed points,

$$
\left.H_{<}^{\prime}\right|_{u=L_{1}}=0
$$

and

$$
\left.H_{>}^{\prime}\right|_{u=L_{2}}=0
$$

This Green's function then determines the metric perturbation via

$$
\begin{aligned}
\bar{h}_{\mu \nu}\left(x, u=L_{1}\right)= & \int d^{4} x_{0} d u_{0} G\left(x, u=L_{1} ; x_{0}, u_{0}\right) \\
& \times\left\{\frac{-e^{2 b u}}{M^{3}}\left[S_{\mu \nu}-\frac{1}{6} \eta_{\mu \nu} S_{\lambda}^{\lambda}\right]\right. \\
& \left.\times \delta\left(u_{0}-L_{2}\right)-\frac{1}{M^{3}} Q_{\mu \nu}\right\} \\
= & \int d^{4} x_{0} d u_{0} G\left(x, u=L_{1} ; x_{0}, u_{0}\right) \\
& \times\left\{\frac{-e^{2 b u}}{M^{3}}\left[S_{\mu \nu}-\frac{1}{6} \eta_{\mu \nu} S_{\lambda}^{\lambda}\right]\right. \\
& \left.\times \delta\left(u_{0}-L_{1}\right)-\frac{1}{M^{3}} Q_{\mu \nu}\right\} .
\end{aligned}
$$

Recall from Eq. (11) that $S_{\mu \nu}=e^{-2 b u} \operatorname{diag}(-\rho, p, p, p)$. Now we define

$$
\underline{W}_{\mu \nu} \equiv \frac{-e^{2 b L_{1}}}{M^{3}}\left[\underline{S}_{\mu \nu}-\frac{1}{6} \eta_{\mu \nu} S_{\lambda}^{\lambda}\right]
$$

and

$$
W_{\mu \nu}(x)=\frac{-e^{2 b L_{2}}}{M^{3}}\left[S_{\mu \nu}(x)-\frac{1}{6} \eta_{\mu \nu} S_{\lambda}^{\lambda}(x)\right] .
$$

Using the Fourier convention

$$
W_{\mu \nu}(x)=\int \frac{d^{4} p}{(2 \pi)^{4}} \widetilde{W}_{\mu \nu}(p) e^{i p \cdot x}
$$

and similarly for $\underline{W}_{\mu \nu}$, we can solve for $\underline{\widetilde{W}}_{\mu \nu}(p)$ as

$$
\widetilde{\widetilde{W}}_{\mu \nu}(p)=\widetilde{W}_{\mu \nu}(p) \frac{H\left(p, u=L_{1}, u_{0}=L_{2}\right)}{H\left(p, u=L_{1}, u_{0}=L_{1}\right)} .
$$

Up to this point, the analysis has been rigorous. To make the result more transparent, we will make some simplifying assumptions which are not necessarily all realistic. However, the qualitative feature that we wish to bring out does not seem to depend upon the unrealistic features of the assumptions. Let us assume that one can write the projected perturbation stress tensor as a perfect fluid of the form

$$
\underline{S}^{\mu \nu}=\left(\underline{p_{2}} \eta^{\mu \nu}+\left(\underline{p_{2}}+\underline{\rho_{2}}\right) U^{\mu} U^{\nu}\right),
$$

where the velocity

$$
U^{\mu} \equiv \frac{d x^{\mu}}{d \tau}
$$

is that of a comoving fluid element and $p_{2}$ and $\rho_{2}$ are scalars (pressure and energy density) measured by an observer in a 
locally inertial frame that moves with the fluid. ${ }^{4}$ We are interested in a nearly static fluid configuration. In that case $U^{i}$ for $i=1,2,3$ can be neglected and the condition

$$
U^{\mu} U_{\mu}=-1
$$

gives

$$
\underline{S}^{00}=\underline{\rho}_{2} e^{-2 b L_{1}}
$$

and

$$
\underline{S}_{\lambda}^{\lambda}=\left(-\underline{\rho_{2}}+3 \underline{p_{2}}\right) e^{-2 b L_{1}}
$$

We remind the reader that underlined quantities refer to projections from brane 2 to brane 1 . Hence, $\rho_{2}$ is the effective perturbation density as seen on brane 1 due to a perturbation density produced on brane 2 .

We seek a relation between the Fourier transform of the density on brane 2 ,

$$
\hat{\rho}_{2}(t, \vec{k})=\int d^{3} x e^{-i \vec{k} \cdot \vec{x}} \rho_{2}(x),
$$

and the Fourier transform of its projection onto brane 1,

$$
\underline{\hat{\rho}_{2}}(t, \vec{k})=\int d^{3} x e^{-i \vec{k} \cdot \vec{x}} \underline{\underline{\rho_{2}}}(x) .
$$

We have

$$
\underline{\hat{\rho}_{2}}(t, \vec{k})=\frac{-6 M^{3}}{5+3 \underline{w_{2}}} \int \frac{d k^{0}}{(2 \pi)} e^{-i k^{0} t} \widetilde{W}_{00}\left(k^{0}, \vec{k}\right)
$$

where we have defined $w_{2} \equiv p_{2} / \rho_{2}$ and assumed it to be independent of time. ${ }^{5}$ If the stress tensor on brane 2 can also be described by a perfect fluid with an approximately constant equation of state of the form $w_{2}=w_{2}$, we find

$$
\underline{\hat{\rho}_{2}}(t, \vec{k})=\int \frac{d k^{0}}{2 \pi} e^{-i k^{0} t} \frac{H\left(k, u=L_{1}, u_{0}=L_{2}\right)}{H\left(k, u=L_{1}, u_{0}=L_{1}\right)} \tilde{\rho}_{2}(k) .
$$

Now, to simplify our results, we shall neglect any $\dot{a} / a(u) \Delta t$ corrections where $\dot{a} / a(u)$ is the spatial expansion rate of 3 -slice at coordinate $u$ and $\Delta t$ is the time difference with respect to some initial time. We will also assume for now that the density perturbation is static, i.e.,

$$
\tilde{\rho}_{2}(k)=\hat{\rho}_{2}(\vec{k})(2 \pi) \delta\left(k^{0}\right)
$$

where the notation "hat" over a quantity refers to a Fourier transformed static quantity (i.e., a function only of 3-vector $\vec{k})$. Hence, we have

\footnotetext{
${ }^{4}$ Note that these projected effectively "Planck brane" densities do show up unscaled in the zero-mode effective theory. Hence, these can be measured by a low energy observer.

${ }^{5}$ This will in general not be true.
}

$$
\underline{\hat{\rho}_{2}}(\vec{k}) \approx \frac{H\left(k^{0}=0, \vec{k}, u=L_{1}, u_{0}=L_{2}\right)}{H\left(k^{0}=0, \vec{k}, u=L_{1}, u_{0}=L_{1}\right)} \hat{\rho}_{2}(\vec{k})
$$

which gives the effective perturbation density on brane 1 due to perturbation density on brane 2 . In particular, if we live on brane 1 , then given a density perturbation generated on a hidden brane identified as brane 2 at $u=L$, we can deduce its amplitude from the point of view of our brane.

\section{Simple example with flat metric}

To gain some intuition for the quantity in Eq. (49), consider a noncompact flat 5D spacetime, $d s^{2}=-d t^{2}+d \vec{x}^{2}$ $+d u^{2}$, which is obtained from the Randall-Sundrum metric of Eq. (5) by setting $b=0$ and removing the boundaries. The differential equation for the case $p^{0}=0$ is

$$
H^{\prime \prime}=\vec{p}^{2} H
$$

We can impose the boundary condition

$$
\begin{gathered}
H_{<}(u \rightarrow-\infty)=0, \\
H_{>}(u \rightarrow \infty)=0,
\end{gathered}
$$

and the jump condition $\left.\left(H_{>}^{\prime}-H_{<}^{\prime}\right)\right|_{u_{0}}=-1$ and $H_{>}\left(u_{0}\right)$ $=H_{<}\left(u_{0}\right)$. Suppose our brane (a probe brane) is located at $u=0$ and the hidden brane at $u=L$. We trivially find

$$
H\left(p^{0}=0, \vec{p}, u=0, u_{0}=L\right)=\frac{e^{-|\vec{p}| L}}{2|\vec{p}|} .
$$

Thus we have

$$
\underline{\hat{\rho}_{2}}(\vec{k})=e^{-k L} \hat{\rho}_{2}(\vec{k}) .
$$

This equation gives us the size of density perturbations [see Eq. (45)] as seen on our brane at $u=0$ in terms of density perturbations $\hat{\rho}_{2}(\vec{k})$ produced on the hidden brane at $u$ $=L$. One can see that $\widehat{\rho_{2}}(\vec{k})$ does not get significant contribution from wavelengths that are much shorter than the distance $L$. Also note that, for all wavelengths longer than $L$, the exponential plays no role and the perturbations on our brane would look just the same as the perturbation on the other brane.

\section{Projection of perturbations in Randall-Sundrum metric}

Let us now return to the RS-type scenario (AdS embedding). Using the boundary conditions in Eqs. (29)-(32) in Eq. (28), we find solutions for $H\left(k, u, u_{o}\right)$. Using these solutions in Eq. (49), we find the amplitude $\hat{\rho}_{2}(\vec{p})$ of lensed density fluctuations projected onto brane 1 , due to the density fluctuations on brane $2, \hat{\rho}_{2}(\vec{p})$.

\section{Observable brane as brane 1 at $u=0$}

First, suppose that the hidden sector brane is brane 2 at $u=L$ while the observable brane is brane 1 at $u=0$ (see for 
example, Ref. [43]). Then we find the amplitude $\hat{\rho}_{2}(\vec{p})$ of lensed density fluctuations projected onto brane 1 , due to the density fluctuations on brane $2, \hat{\rho}_{2}(\vec{p})$ :

$$
{\hat{\rho_{2}}}_{(}(\vec{p})=\frac{e^{-3 b L}}{\frac{p}{b}\left[I_{2}\left(\frac{p}{b}\right) K_{1}\left(\frac{p}{b} e^{b L}\right)+I_{1}\left(\frac{p}{b} e^{b L}\right) K_{2}\left(\frac{p}{b}\right)\right]} \hat{\rho}_{2}(\vec{p})
$$

where $I_{\nu}(z)$ and $K_{\nu}(z)$ are modified Bessel function of the first and second kind. In the limit that $(p / b) e^{b L} \ll 1$, we have

$$
\lim _{p \rightarrow 0} \hat{\rho}_{2}(\vec{p})=e^{-4 b L} \hat{\rho}_{2}(\vec{p})
$$

while in the limit that $(p / b) e^{b L_{\gg}} \gg 1$, we have

$$
\underline{\hat{\rho}_{2}}(\vec{p}) \sim 2 e^{-5 b L / 2} e^{(-p / b)\left(e^{b L}-1\right)} \hat{\rho}_{2}(\vec{p}) .
$$

Equations (55), (56), (57) are key results which answer our original question for the Randall-Sundrum case: given a (Fourier transformed, static) density perturbation $\hat{\rho}_{2}(\vec{p})$ on brane 2 , these equations tell us the amplitude $\hat{\rho}_{2}(\vec{p})$ of the lensed perturbations as seen by brane 1 .

We have obtained the following important result: perturbations intrinsic to the hidden brane appear to be reduced in amplitude by an exponential factor. One can understand the $e^{-4 b L}$ dilution in the long wavelength limit as follows. First, the density simply scales as the spatial volume. Since the volume at $u=0$ is larger by $e^{3 b L}$ on our brane, the density is reduced by the inverse of this factor. Second, in addition to the spatial volume dilution, the time interval is increased in projecting onto the Planck brane. Hence, the energy is redshifted by a factor $e^{b L}$ such that the energy density is reduced by this additional factor, giving a total of $e^{-4 b L}$ for the potential. As a result, perturbations as seen by our brane are reduced by an overall factor of $e^{-4 b L}$.

In Sec. II A, we discussed that $\rho_{2}$ cannot be measured from low energy measurements (measurements that do not resolve the bulk); one must derive an effective 4D observable describing a low energy observer's point of view. What we have just described from a 5D point of view is precisely this $4 \mathrm{D}$ observable, and it is in agreement with Sec. II A in the long wavelength limit.

This result leads to the interesting possibility that large amplitude perturbations produced on brane 2 may still satisfy Eq. (1) and serve as a mechanism for generation of density perturbations on our brane.

One might hope to solve the flatness problem due to this large suppression factor. If the $e^{-4 b L}$ suppression is sufficiently strong, one might naively suspect that lensed perturbations of very small amplitude might be able to smooth out initially large perturbations on brane 1 . However, as we will discuss later, this idea fails.

Because of reciprocity, small fluctuations on brane 1 can imprint large perturbations elsewhere. Even in scenarios without a second brane, as in RSII, one might fear that small fluctuations on our brane will appear large as one moves farther and farther from our brane and give rise to back reactions. (For related work, see [29,30,32,33].) However, as was shown in Ref. [29] and as is reviewed in the Appendix, there is not necessarily a large back reaction because the higher momentum modes can still be suppressed. The degree to which there is a back reaction depends upon the degree of spatial localization of the perturbation. Roughly speaking, the less localization leads to a greater back reaction. Further discussions of these issues can be found in the Appendix.

Our primary result for this section is that large amplitude perturbations produced on brane 2 (from a 5 dimensional point of view) may look suppressed from the point of view of brane 1, such that they satisfy Eq. (1) and hence can provide seeds for structure formation in our observable universe.

\section{Our observable brane as brane 2 at $u=L$}

The RSI scenario attempts to solve the hierarchy problem with an inverse warping of the one we have been studying above: hence we will now consider the observable sector at $u=L$ and the hidden sector at $u=0$. Any long wavelength density perturbations produced in the extra dimensions may be exponentially enhanced from the point of view of a high energy observer on our brane. We have

$$
\begin{aligned}
& \frac{H\left(k^{0}=0, \vec{k}, u=L, u_{0}=0\right)}{H\left(k^{0}=0, \vec{k}, u=L, u_{0}=L\right)} \\
& =\frac{e^{2 b L}}{\frac{p}{b}\left[I_{2}\left(\frac{p}{b} e^{b L}\right) K_{1}\left(\frac{p}{b}\right)+I_{1}\left(\frac{p}{b}\right) K_{2}\left(\frac{p}{b} e^{b L}\right)\right]} .
\end{aligned}
$$

In the limit $(p / b) e^{b L} \ll 1$, this goes to

$$
\lim _{p \rightarrow 0} \frac{H\left(k^{0}=0, \vec{k}, u=L, u_{0}=0\right)}{H\left(k^{0}=0, \vec{k}, u=L, u_{0}=L\right)}=e^{4 b L} .
$$

Hence we find in the long wavelength limit

$$
\widehat{\rho_{1}}(\vec{p})=e^{4 b L} \hat{\rho}_{1}(\vec{p})
$$

where consistently as before $\widehat{\rho_{1}}$ is the projected density onto $u=L$ due to a source at $u=\overline{0}$ (at brane 1 ).

However, as noted in Sec. II A, from a low energy observer's point of view (with the radion stabilized) who sees only the massless Kaluza-Klein (KK) mode, the enhancement is invisible: the enhancement of the effective density is canceled by the warp factor such that the effective gravity sees exactly the original energy density of the Planck brane. (This has already been noted by Ref. [42].) One can see this from Eq. (22):

$$
\bar{\rho}^{(4 D)} \sim \hat{\rho}_{1}=e^{-4 b L}\left(\hat{\rho}_{1} e^{4 b L}\right)=e^{-4 b L}\left(\hat{\rho}_{1}\right) .
$$


Here, we have used Eq. (60) in the last equality. Indeed the first and last expressions in Eq. (61) satisfy Eq. (21) for the four dimensional effect of a source at $u=L$ (although $\hat{\rho}_{1}$ here really is an effective source).

The matter on the hidden sector brane looks like dark matter to us, which can be constrained from observations. Even though from a low energy observer's point of view, the lensed factor is canceled, given that the natural scale on the Planck brane is the Planck scale, mechanisms suppressing Planckian densities are necessary to have a phenomenologically acceptable model. Here we have a hierarchy problem: we have no explanation for the fact that the localized density on our observable brane is exponentially smaller than the localized density on the hidden sector Planck brane. We refer the reader to Ref. [42] for more discussion on this.

\section{Scale of perturbations}

We return to the scenario with our observable brane at $u$ $=0$ (brane 1). It may seem that, even without Lorentz violation in the bulk (see e.g. [7,13]), the perturbations on brane 2 can lead to apparently acausal perturbations to be projected onto our brane. Because of the relationship

$$
p_{\text {phys } 2}=e^{b L} p_{\text {phys } 1}
$$

for a fixed coordinate momentum vector, short wavelength perturbations on brane 2 can look like long wavelength perturbations on brane 1 . However, for a cosmological scale factor independent of $u$, i.e.

$$
d s^{2}=e^{-2 b u}\left(-d t^{2}+a^{2}(t) d \vec{x}^{2}\right)+d u^{2}
$$

the physical horizon size is also larger by the same factor on brane 1. Hence perturbations generated on causal scales (smaller than the horizon) on brane 2 remain on causal scales on brane 1 . This fact is a reflection of the fact that this metric is conformal to Friedmann-Robertson-Walker with an extra dimension $^{6}\left(-d t^{2}+a^{2} d \vec{x}^{2}+d z^{2}\right)$, and that conformal transformations leave invariant the causal structure of the metric.

The size of the comoving horizon is determined by setting $d s^{2}=0$ such that we find $d_{\text {comoving }}=\int_{0}^{t}\left[d t^{\prime} / a\left(t^{\prime}\right)\right]$. The size of the physical horizon is then

$$
d_{H}(u, t)=e^{-b u} a(t) \int_{0}^{t} \frac{d t^{\prime}}{a\left(t^{\prime}\right)} .
$$

Thus on brane 1 at $u=0$, the horizon size is $d_{H}(u=0, t)$ $=a(t) \int_{0}^{t}\left[d t^{\prime} / a\left(t^{\prime}\right)\right]$, while on brane 2 at $u=L$, the horizon size is $d_{H}(u=L, t)=e^{-b L} a(t) \int_{0}^{t}\left[d t^{\prime} / a\left(t^{\prime}\right)\right]$. Indeed the physical horizon is larger on brane 1 by

$$
d_{H}(u=0, t)=e^{b L} d_{H}(u=L, t),
$$

the same factor as in Eq. (62). Both the wavelength of the perturbation and the horizon are shifted by the same amount.

\footnotetext{
${ }^{6}$ There is no acausality in a Friedmann-Robertson-Walker (FRW) metric.
}

Hence, with any metrics which maintain $S O(1,3)$ isometry (neglecting the time dependence of the scale factor), including the RS metrics, one cannot induce perturbations on scales beyond the horizon length from perturbations below the horizon length. In the next section we consider a scenario in which $S O(1,3)$ is violated by the warping and hence acausal perturbations can be generated.

Finally, note that from Eq. (58) for the situation where we live on brane 2 and the perturbation is on brane 1 (e.g. RSI), the brane 1 perturbation wavelength must be longer than $e^{b L} / b$ to show up as unsuppressed perturbation on our brane with a wavelength longer than $1 / b$.

\section{DENSITY PERTURBATIONS IN SCENARIOS WITH "SHORTCUT GEODESICS"}

Reference [7] proposed a non-inflationary solution to the cosmological horizon problem in braneworlds. A signal traveling along an extra-dimensional null geodesic may leave our three-brane, travel into the extra dimensions, and subsequently return to a different place on our three-brane in a shorter time than the time a signal confined to our threebrane would take. Hence, these geodesics may connect distant points which would otherwise be "outside" the four dimensional horizon (points not in causal contact with one another). Such shortcut metrics break $S O(1,3)$ isometry along the coordinate directions parallel to the 3-brane; i.e. these metrics explicitly break Lorentz isometry.

Here we discuss the possibility that perturbations created elsewhere on small length scales may be lensed to appear on our brane on apparently acausal superhorizon length scales. In other words, not only may there be an explanation of large scale smoothness via these shortcut metrics, but also an explanation of perturbations on superhorizon scales.

Following [7], we consider the Lorentz violating spacetime [without $S O(1,3)$ isometry],

$$
d s^{2}=-d t^{2}+e^{-2 b u} d \vec{x}^{2}+d u^{2} .
$$

We perturb about this background spacetime as

$$
\begin{aligned}
d s^{2}= & d u^{2}-d t^{2}\left[1+h_{00}(x, u)\right]+e^{-2 b u}\left[\delta_{i j}+h_{i j}(x, u)\right] d x^{i} d x^{j} \\
& +2 e^{-b u} h_{0 i} d x^{i} d t
\end{aligned}
$$

If we assume that the gravity interaction is pointlike, the equation of motion can be read off from Ref. [44] and is

$$
\frac{1}{\sqrt{g}} \partial_{A}\left[\sqrt{g} g^{A B} \partial_{B}\right] h_{00} \sim \frac{-A}{M^{3}} \rho_{2} \delta(u-L)
$$

where $A$ is a constant coefficient of order 1 which depends on the equation of state. We can use the same Green's function formalism as before. We find the general homogeneous solution with $p^{0}=0$ to be

$$
\begin{aligned}
H(p, u, L)= & {\left[c_{1}-c_{2} z(u)\right] \cosh [z(u)] } \\
& +\left[c_{2}-c_{1} z(u)\right] \sinh [z(u)]
\end{aligned}
$$

where 


$$
z(u)=\frac{p}{b} e^{b u}
$$

with $p \equiv|\vec{p}|$, and $c_{1}$ and $c_{2}$ are constants to be specified by the boundary conditions. Imposing the boundary conditions, we have

$$
\begin{aligned}
H_{<}\left(p^{0}=\right. & \left.0, \vec{p}, u, u_{0}=u_{0}\right) \\
= & \frac{-e^{-3 b u_{0}}}{p^{3} \sinh \left[\frac{p}{b}\left(1-e^{b L}\right)\right]}\left(p e^{b u_{0}} \cosh \left[\frac{p}{b}\left(e^{b L}-e^{b u_{0}}\right)\right]\right. \\
& \left.+b \sinh \left[\frac{p}{b}\left(e^{b L}-e^{b u_{0}}\right)\right]\right)\left(p e^{b u} \cosh \left[\frac{p}{b}\left(1-e^{b u}\right)\right]\right. \\
& \left.+b \sinh \left[\frac{p}{b}\left(1-e^{b u}\right)\right]\right)
\end{aligned}
$$

and

$$
\begin{aligned}
H_{>}\left(p^{0}=\right. & \left.0, \vec{p}, u, u_{0}=u_{0}\right) \\
= & \frac{-e^{-3 b u_{0}}}{p^{3} \sinh \left[\frac{p}{b}\left(1-e^{b L}\right)\right]}\left(p e^{b u} \cosh \left[\frac{p}{b}\left(e^{b L}-e^{b u}\right)\right]\right. \\
& \left.+b \sinh \left[\frac{p}{b}\left(e^{b L}-e^{b u}\right)\right]\right)\left(p e^{b u_{0}} \cosh \left[\frac{p}{b}\left(1-e^{b u_{0}}\right)\right]\right. \\
& \left.+b \sinh \left[\frac{p}{b}\left(1-e^{b u_{0}}\right)\right]\right) .
\end{aligned}
$$

These expressions can be used as before to yield

$$
\underline{\hat{\rho}_{2}}(\vec{p})=\frac{e^{-2 b L}}{\cosh \left[\frac{p}{b}\left(e^{b L}-1\right)\right]+\frac{1}{(p / b)} \sinh \left[\frac{p}{b}\left(e^{b L}-1\right)\right]} \hat{\rho}_{2}(\vec{p})
$$

Again, underlined quantities refer to the fictitious effective quantities on brane 1 . Given a density perturbation $\hat{\rho}_{2}(\vec{p})$ on brane $2, \hat{\rho}_{2}(\vec{p})$ is the lensed perturbation seen by observers on brane $\overline{1}$.

Note that the perturbation is exponentially suppressed. In the limit that $(p / b) e^{b L} \ll 1$, we have

$$
\lim _{p \rightarrow 0} \hat{\rho}_{2}(\vec{p}) \sim e^{-3 b L} \widehat{\rho_{2}}(\vec{p})
$$

while in the limit that $(p / b) e^{b L} \gg 1$, we have

$$
\underline{\hat{\rho}_{2}}(\vec{p}) \sim e^{-2 b L} e^{-(p / b)\left(e^{b L}-1\right)} \hat{\rho}_{2}(\vec{p})
$$

The $e^{-3 b L}$ suppression for the long wavelength case is easy to understand as before. Any energy density on the Planck brane is diluted by the volume factor.
Let us now discuss the scale of the perturbations for the shortcut metric background. Since on brane 2 the momentum vector is enhanced by

$$
p_{\text {phys } 2}=e^{b L} p_{\text {phys } 1},
$$

short wavelength perturbations on brane 2 can look like long wavelength perturbations on brane 1 . Here the size of the comoving horizon, obtained by setting $d s^{2}=0$, is $d_{\text {comoving }}$ $=e^{b u} \int d t$. Then the size of the real (proper) horizon is $d_{H}$ $=e^{-b u} d_{\text {comoving }}$, such that $d_{H}=\int d t$ and is independent of $u$. Hence perturbations created on causal length scales on brane 2 can appear on much larger superhorizon length scales on brane 1 .

Suppose brane 2 corresponds to the hidden sector brane while brane 1 corresponds to the observable sector brane. Small scale density fluctuations produced elsewhere in the universe appear on our brane as large scale fluctuations of smaller amplitude. This scenario may thus both potentially explain the large scale smoothness of the universe (the horizon problem) as well as explain the superhorizon small density perturbations required to provide seeds for large scale structure. Of course, there still remains a significant challenge in identifying a physical system that generates the appropriate $S O(1,3)$ isometry breaking warped background.

\section{DISCUSSION}

We have found that perturbations may be lensed by a warped geometry, such that both their scale and amplitude may look different to an observer on our brane than where they were originally produced. We have found that fluctuations can look large or small because of the volume factors. For example, if the volume on our brane is larger than on a hidden brane for a fixed coordinate volume, large perturbations on a hidden brane may appear to be small perturbations on our observable brane due to a warping of the geometry. The most interesting application of lensed perturbations is to "shortcut metrics" [7] in which geodesics traverse the extra dimensions. We found that large amplitude perturbations of small wavelength on one brane can be lensed to be small amplitude perturbations of superhorizon length scales here as is required for structure formation. Hence shortcut metrics may provide an alternative to inflation with two important features: a possible solution to the horizon problem and a mechanism to generate perturbations necessary for structure formation.

Suppose the perturbations on large scale are negligible for matter confined to our brane, i.e., $\delta \rho_{0}=0$, where the subscript 0 denotes our observable brane confined fields. The quantity of interest is

$$
\frac{\delta \rho_{t o t}}{\rho_{t o t}}=\frac{\delta \rho_{h}}{\rho_{0}+\rho_{h}},
$$

where $\rho_{\text {tot }}$ includes the energy density $\rho_{0}$ intrinsic to our observable universe, and the underline as before indicates the projection onto our brane. If $\rho_{h} \ll \rho_{0}$, then the amplitude of $\delta \rho_{\text {tot }} / \rho_{\text {tot }}$ can be very much smaller than $\delta \rho_{h} / \rho_{h}$, the origi- 
nal amplitude of the perturbations on the hidden brane (subscript $h$ ) where they were produced.

In addition, for the case of Lorentz violating metrics such as the shortcut metrics, there is an apparent acausal behavior of the perturbation. As the scale of the perturbations can be stretched, this hidden sector "causal" perturbation can contribute significantly to the superhorizon ("acausal") perturbations that are required to explain the large scale structure of our universe and the peaks in the microwave background [45].

Above we have focused on a 2 brane situation. However, our results apply equally to a single brane. For example, density fluctuations produced in the bulk will have the same projection on our brane (as from a hidden brane) and may thus be interesting for structure formation or for providing constraints.

Even if our brane does have an inflationary period to solve the flatness and the horizon problem, lensed perturbations may be essential for structure formation if the inflation itself produces perturbations that are too small (e.g., if the height of the inflaton potential is too low), although the final form of the gravitational collapse is highly model dependent (time dependence of the background warping). In that case generation of perturbations elsewhere, such as on a hidden brane, that are propagated to our brane in the way described in the paper, may be essential to the formation of structure in the universe. In fact perturbations generated in the bulk would carry the same exponential suppression factors we have been describing. However, for the lensed perturbations to serve this purpose, the causal perturbations must be scale invariant. We close this paper by presenting some ideas for future studies.

\section{A. Generation of density perturbations}

Although we leave the generation of scale invariant causal perturbations to future studies, here we speculate about possible origins of perturbations on the hidden brane. One possible mechanism for the generation of scale invariant density fluctuations on the hidden brane would be a second order phase transition near criticality, where the order parameter field has scale invariant two point correlations. Another possible mechanism for generation of scale invariant fluctuations is when a hidden brane has a period of inflation, say for a few $e$-foldings, during which very large perturbations, $\delta \rho_{h} / \rho_{h} \sim 1$ are produced. While it is not much harder to get a few efoldings than it is to get 60 , the advantage here is that the potential does not need to be fine-tuned to be flat. If this inflation is due to a potential, then it is the height of the potential that determines the amplitude of the density fluctuations. In ordinary inflationary scenarios, the ratio of the height of the potential to the (width) ${ }^{4}$ must be very small, less than $\sim\left(10^{-8}\right)[3]$; this small ratio usually requires a fine-tuned small parameter. On the hidden brane, on the other hand, since the amplitude of the fluctuations can be as large as we like, the height of the potential can be anything. Then the potential does not need to be flat and there do not need to be any small parameters. For example, the width and height of the potential may both be of order the grand unification scale $M_{G U T}$. If there are many branes sitting in the warped extra dimensions, then as long as one of them generates large perturbations e.g. by having a brief inflationary period, then we will feel the existence of these perturbations and they will appear to us to be much smaller in amplitude.

Of course, the flip side of this is that for this causally induced acausal perturbation scheme to work, the scale invariance must not be disturbed during the time structure formation commences. In other words, not only must the scale invariant perturbations be set up, it must be protected until structure formation is well under way. This seems to be a significant model building challenge. In particular, the study of this issue will depend on how the transition (if any) from the asymmetrically warped metric to a non-asymmetrically warped metric takes place.

We have not investigated the time evolution of the lensed perturbations. Our perturbation equations are valid at best over a time interval much smaller than $1 / H$, where Hubble constant $H$ is the expansion rate of the universe. A study of the time evolution of the perturbations would require more model dependent investigations by perturbing about fully time dependent backgrounds. Some work in braneworld density perturbations in time dependent backgrounds can be found in [35-41]. We also have not investigated how 4D gravity will be recovered after these perturbations are generated. Because the Lorentz asymmetry in warping needs to be strong, 4D effective theory description probably will not be valid in describing the transition process.

\section{B. Flatness}

Here we briefly describe an attempt to solve the flatness problem due to these lensed perturbations. One statement of the flatness problem is that there are fluctuations in density on our brane that give rise to too much curvature. One might hope to smooth out large fluctuations on our brane by somehow "averaging" them with small fluctuations appearing from some hidden brane(s). As we will argue, one finds that the amplitude of perturbations on our brane can be reduced by at most $1 / \sqrt{2}$ due to the effects from one hidden brane, or $1 / \sqrt{N}$ where $N$ is the number of hidden branes.

Let us first consider a scenario with two branes: our observable brane 1 and a hidden brane 2 . We begin with a conserved stress tensor of a perfect fluid on each of the branes. When we perturb the fluid with small fluctuations, we find that the system looks like a set of coupled oscillator equations; the warping of the geometry plays a role in that it enters in the mechanical tension. One might hope that small vibrations of brane 2 might absorb vibrations of brane 1, i.e., that small perturbations projected from the hidden brane might reduce large perturbations on our observable brane. However, we find that the reduction of perturbations on our brane is not significant. In fact, equipartition of energy tells us that on the average the largest amount of energy that can be lost by our system 1 is half of the original energy. Thus vibrations of brane 1 can be reduced by at most $1 / \sqrt{2}$ on the average. 
If there are $N$ hidden branes instead of just one, then equipartition of energy implies that the amplitude of perturbations on our brane can be reduced by at most $1 / \sqrt{N}$. In addition, our brane must be sitting at a special place in the geometry, such that the direction of lensing of the perturbations from the other branes makes them all appear small to us.

Hence, even though a number of attempts have been made in the context of extra dimensions to find an alternative mechanism for solving the flatness problem [46], the usual inflationary scenario seems most robust, thus far. Ekpyrotic scenarios [47] use the flatness of BPS branes. However, this approach begins with a particular choice of initial conditions rather than providing a dynamical explanation of the flatness of our universe. (Still, of course there may be quantum cosmological mechanisms that drive the amplitude to be peaked in such configurations.)

On the other hand, braneworld extra dimensions may make the flatness problem worse [13]. ${ }^{7}$ Reference [13] first pointed out a new signature for the existence of extra dimensions in the context of braneworld scenarios: due to the "nonflatness" of the bulk, gravitational waves may travel at different speeds than photons from sources. (See Ref. [19] for a related perspective and Ref. [50] for earlier reference to this phenomenon.) Then if one were to detect gravitational waves and find no time difference between arrival times of photons and gravitational waves, this detection would present yet another aspect of the cosmological flatness problem.

\section{Speculation on dark energy}

We end with one last comment. We have shown that, in extra dimensions with warped geometries, density perturbations appear to have different amplitudes on our brane than where they were originally produced. Hence, it is tempting to speculate that large energy densities with a negative equation of state on the hidden sector brane may be projected to a small energy density with a negative equation of state on our brane, thereby explaining the current acceleration of the universe indicated most dramatically by supernova observations $[51,52]$. An attempt to explain the acceleration in terms of an energy density requires the energy density to be small. While the generation of such a small value on our brane typically requires fine-tuning, lensing of a larger value generated elsewhere may suggest an improvement. This question deserves further investigation.

\section{ACKNOWLEDGMENTS}

We thank Csaba Csaki, Lisa Everett, Arthur Hebecker, Rocky Kolb, Jianxin Lu, Antonio Riotto, Riccardo Rattazzi, Ren-Jie Zhang, and particularly Robert Brandenberger, Jim Liu, and John March-Russell for useful conversations. We acknowledge support from the Department of Energy via the

\footnotetext{
${ }^{7}$ For an example of a resolution, see Refs. [48,49].
}

University of Michigan. K.F. thanks Columbia University for hospitality during her visit.

\section{APPENDIX: BACK REACTION}

Based on our work in the body of the paper, one might naively expect a reverse lensing effect: if large density perturbations on a hidden brane appear to be small on our brane, then one might fear that small density perturbations on our brane might appear larger and larger as one goes out to large distances, eventually leading to back reactions. However, we show here that, for point sources on our brane, factors cancel each other out such that this is not the case. We find agreement on this point with [29]. On the other hand, we show that for non-pointlike sources, larger back reaction is expected.

Below we first consider a point source and then an extended source on our brane; both are assumed to be static. We ask the question: what is the resulting gravitational potential at large distances? We find that 4D momentum near zero mode contributions are exponentially enhanced but all higher momentum modes are suppressed. For the point source, when one adds up the contributions from all modes to invert the Fourier transform, one finds that the number of exponentially enhanced modes form an exponentially small set, and hence the sum is not exponentially enhanced. However, as the source becomes increasingly delocalized (less pointlike) the exponentially enhanced zero mode contributions becomes increasingly important. Hence, the back reaction is increasingly significant for less localized density perturbations. This is related to the fact that for infinite charge distributions of codimension one objects, the energy associated with brining in a test charge from infinity is infinite.

Let us now show the mathematical details of this result. Equation (9), the linearized field equation about the AdS metric, can be rewritten as

$$
\begin{aligned}
\frac{1}{\sqrt{g}} \partial_{a}\left[\sqrt{g} g^{a b} \partial_{b}\right] \tilde{h}_{r q}= & \frac{1}{f}\left[\tilde{h}_{\mu r}^{\mu}\left(u_{i}\right)+\tilde{h}_{\mu q r}^{\mu}\left(u_{i}\right)-\tilde{h}\left(x, u_{i}\right)_{, r q}\right. \\
& -\int_{u_{i}}^{u} d u^{\prime \prime} \frac{R}{3 M^{3}} T_{55, r q}+f^{\prime} \eta_{r q} \frac{R}{6 M^{3}} T_{55} \\
& \left.-\frac{1}{M^{3}}\left(T_{q r}-\frac{1}{3} g_{q r} T_{s}^{s}\right)\right]
\end{aligned}
$$

where $f(u) \equiv e^{-2 u / R}, R$ is the radius of curvature for the AdS space, $T_{q r}$ is the 5D stress energy tensor, and the tildes indicate the perturbation is calculated in a particular gauge in which fictitious bulk instabilities due to nonlocal sources are removed. The first three terms on the right hand side correspond to the initial conditions, and the terms involving $T_{55}$ are gauge dependent. The Green's function for this system has been calculated in an earlier section of our paper in the form 


$$
G\left(x, u ; x_{0}, u_{0}\right)=\int \frac{d^{4} p}{(2 \pi)^{4}} e^{i p \cdot\left(x-x_{0}\right)} H\left(p, u, u_{0}\right)
$$

Let us focus on the situation where a localized source on the Planck brane seems to give a large back reaction far away in the bulk. We found

$$
\begin{aligned}
H(p, L, 0) & =H_{>}(p, L, 0) \\
& =\frac{b e^{b L}}{p^{2}\left[I_{1}\left(e^{b L} \frac{p}{b}\right) K_{1}\left(\frac{p}{b}\right)-I_{1}\left(\frac{p}{b}\right) K_{1}\left(e^{b L} \frac{p}{b}\right)\right]}
\end{aligned}
$$

where $>$ symbol indicates that this Green's function is only valid for the domain for which

$$
L>0 \text {. }
$$

Now we take as our starting point: given a point source on the brane at $u=0$, what behavior does one find at large distances? We take the localized source at $u=0$ : i.e.

$$
T_{00} \propto m \delta(u) \delta^{(3)}\left(x-x_{0}\right)
$$

where $m$ is the mass. Accounting for the contribution only from the sources localized to the brane in the bulk, we can write the associated $\widetilde{h}_{00}$ as

$$
\tilde{h}_{00}\left(x_{0}, u=L\right)=\left.c_{3} m \int \frac{d^{3} p}{(2 \pi)^{3}} H_{>}(p, L, 0)\right|_{p_{0}=0}
$$

where $c_{3}$ is a constant. Let us try to estimate this integral. First, we expand $p^{2} H_{>}$to second order in $p$ to obtain

$$
\begin{aligned}
p^{2} H_{>}(p, L, 0)= & \frac{2 b e^{2 b L}}{\left(e^{2 b L}-1\right)}+p^{2}\left[\frac{-e^{6 b L}+4 b L e^{4 b L}+e^{2 b L}}{4 b\left(e^{2 b L}-1\right)^{2}}\right] \\
& +O\left(p^{4}\right) .
\end{aligned}
$$

Note that

$$
\lim _{p \rightarrow 0} H_{>}(p, L, 0) \sim \frac{2 b}{p^{2}} .
$$

We can expand this to leading order in $e^{b L}$ to obtain

$$
p^{2} H_{>}(p, L, 0)=2 b-p^{2}\left(\frac{e^{2 b L}}{4 b}-L+\frac{1}{2 b}\right) .
$$

From the fact that $H_{>}(p, L, 0)$ is positive definite and rapidly falling, we can approximate Eq. (A1) by integrating up to

$$
p_{\max }=\frac{2 \sqrt{2} b}{\sqrt{e^{2 b L}-4 b L+2}},
$$

resulting in

$$
\begin{aligned}
\tilde{h}_{00}\left(x_{0}, u\right. & =L) \approx \frac{8 c_{3} m b^{2}}{6 \pi^{2} \sqrt{\frac{1}{2} e^{2 b L}-2 b L+1}} \\
& \approx \frac{8 \sqrt{2} c_{3} m b^{2} e^{-b L}}{6 \pi^{2}}+O\left(e^{-2 b L}\right)
\end{aligned}
$$

which indeed drops off as $e^{-b L}$. We find that there is no large back reaction in this case. Note that this result has been obtained earlier by Ref. [29].

Now that we have obtained this result, it remains for us to reconcile it with our previous naive conclusion that density perturbations are magnified by an exponential factor far away from the source. Apparently, to reconcile our naive result with the exponentially reduced potential in the brane at $u=L$ (in the previous paragraph), one needs to introduce an effective energy density at $u=L$ that is exponentially larger. Let us try to see why that is. For the mode with zero 4D momentum, we have the mode equation

$$
\tilde{h}_{00,55}+2 \frac{f^{\prime}}{f} \tilde{h}_{00,5}=\frac{-1}{M^{3} f} T_{00} \sim \frac{-\rho_{1} \delta(u)}{M^{3}} .
$$

The friction term gives an enhancement factor for the $\widetilde{h}_{00}$ contribution from the $u$ dependence; i.e., if the source initially is at $u=0$ and we are evaluating $\tilde{h}_{00}$ at $u=L$, the source at $u=0$ will be amplified as it is propagated to $u$ $=L$. To see this, note that since $T_{00}$ is localized to $u=0$, the zero $4 \mathrm{D}$ momentum mode equation is solved in the bulk by

$$
\widetilde{h}_{00}(k=0)=e^{4 b u} j(x)
$$

for some function $j(x)$ independent of $u$. Hence, we see that the total enhancement of the zero mode in propagating from $u=0$ to $u=L$ is $e^{4 b L}=1 / f^{2}(L)$. Therefore, there needs to be an enhancement of $1 / f(L)^{2}$ in the fictitious source $T_{q r}$. This is precisely what we obtained in the general propagator analysis in an earlier section.

However, as we saw in the analysis at the beginning of this appendix, the enhancement of the zero 4D momentum mode does not mean that the source at $u=0$ is giving a large back reaction far away from the source. The resolution of the apparent paradox is as follows. The configuration space $\widetilde{h}_{q r}$ need not be large even though some Fourier amplitudes are; the amplitude drops off rapidly away from the zero momentum vector due to the fact that gravity is not confined to the brane. To be more explicit, although Eq. (A2) is exponentially larger than $H_{>}(0, L, L)$, it has a phase space of $p_{\max }^{3}$ $\sim 10 b^{3} e^{-3 b L}$ which is exponentially suppressed by a larger exponent than the exponential enhancement coming from $H_{>}(0, L, 0)$. In other words, $H_{>}(0, L, 0) / H_{>}(0, L, L)=e^{4 b L}$ but $\left.\quad \tilde{h}_{00} \propto \int\left[d^{3} p /(2 \pi)^{3}\right] H_{>}(p, L, 0)\right|_{p_{0}=0} \sim H_{>}(0, L$, $\left.p_{\text {max }}\right) p_{\text {max }}^{3} \propto e^{-b L}$. Hence, even with the Fourier transform of the zero 4D momentum mode large, there is no necessarily large back reaction. The zero 4D momentum mode hav- 
ing a large amplitude means that density perturbations on length scales larger than $b^{-1} e^{b L}$ do receive an exponential enhancement.

We have shown above that, contrary to our naive expectations, point sources do not necessarily produce back reactions. However, it remains to consider extended sources. Note that Eq. (A1) valid for a pointlike source can be easily generalized to a spatially smooth energy density:

$$
\tilde{h}_{00}\left(x_{0}, u=L\right)=\left.\int \frac{d^{3} p}{(2 \pi)^{3}} H_{>}(p, L, 0)\right|_{p_{0}=0} \xi\left(x_{0}, \vec{p}\right)
$$

where

$$
\xi(x, \vec{p}) \propto e^{i \vec{p} \cdot \vec{x}} \int d^{3} x^{\prime} \rho\left(\vec{x}^{\prime}\right) e^{-i \vec{p} \cdot \vec{x}^{\prime}}
$$

and $\rho(\vec{x})$ is a smooth 4D energy distribution confined to the brane at $u=0$. Since we have seen that the exponential suppression of $\tilde{h}_{00}$ derived from the small phase space integral about $\vec{p}=0$, we see if $\xi$ is sufficiently peaked about $\vec{p}=0$ (delocalized source), we can naively obtain a large back reaction. A more careful treatment of this issue with the time dependence included deserves further investigation.
[1] C. Bennett et al., Astrophys. J. 434, 587 (1994).

[2] A. Guth, Phys. Rev. D 23, 347 (1981).

[3] F. Adams, K. Freese, and A. Guth, Phys. Rev. D 43, 965 (1991).

[4] K. Freese, J.A. Frieman, and A.V. Olinto, Phys. Rev. Lett. 65, 3233 (1990).

[5] L. Randall and R. Sundrum, Phys. Rev. Lett. 83, 3370 (1999).

[6] L. Randall and R. Sundrum, Phys. Rev. Lett. 83, 4690 (1999).

[7] D. J. Chung and K. Freese, Phys. Rev. D 62, 063513 (2000).

[8] M. A. Clayton and J. W. Moffat, Phys. Lett. B 460, 263 (1999).

[9] M. Visser, Phys. Lett. 159B, 22 (1985).

[10] G. Kalbermann and H. Halevi, "Nearness through an extra dimension," gr-qc/9810083.

[11] H. Ishihara, Phys. Rev. Lett. 86, 381 (2001).

[12] H. Ishihara, Phys. Rev. D 66, 023513 (2002).

[13] D. J. Chung, E. W. Kolb, and A. Riotto, Phys. Rev. D 65, 083516 (2002).

[14] R.R. Caldwell and D. Langlois, Phys. Lett. B 511, 129 (2001).

[15] A.C. Davis, C. Rhodes, and I. Vernon, J. High Energy Phys. 11, 015 (2001).

[16] G. Kalbermann, Int. J. Mod. Phys. A 15, 3197 (2000).

[17] A. Kehagias and E. Kiritsis, J. High Energy Phys. 11, 022 (1999).

[18] P. F. Gonzalez-Diaz, "Cosmological predictions from the Misner brane," hep-th/0008193.

[19] C. Csaki, J. Erlich, and C. Grojean, Nucl. Phys. B604, 312 (2001).

[20] B. Grinstein, D. R. Nolte, and W. Skiba, Phys. Rev. D 63, 105016 (2001).

[21] C. Grojean, F. Quevedo, I. Zavala C., and G. Tasinato, J. High Energy Phys. 08, 005 (2001).

[22] D. Youm, Mod. Phys. Lett. A 16, 2371 (2001).

[23] S. L. Dubovsky, J. High Energy Phys. 01, 012 (2002).

[24] D. Youm, Phys. Rev. D 63, 125011 (2001).

[25] D. Youm, Phys. Rev. D 64, 085011 (2001).

[26] D. Youm, Phys. Rev. D 65, 025008 (2002).

[27] D. Youm, "A varying-alpha brane world cosmology," hep-th/0108237.

[28] C. Deffayet, G. R. Dvali, G. Gabadadze, and A. Lue, Phys. Rev. D 64, 104002 (2001).
[29] S.B. Giddings, E. Katz, and L. Randall, J. High Energy Phys. 03, 023 (2000).

[30] S. B. Giddings and E. Katz, J. Math. Phys. 42, 3082 (2001).

[31] A. Hebecker and J. March-Russell, Nucl. Phys. B608, 375 (2001).

[32] A. Chamblin, S.W. Hawking, and H.S. Reall, Phys. Rev. D 61, 065007 (2000).

[33] H. Lu and C.N. Pope, Nucl. Phys. B598, 492 (2001).

[34] C. Csaki, M. Graesser, C. Kolda, and J. Terning, Phys. Lett. B 462, 34 (1999).

[35] C. van de Bruck and M. Dorca, "On cosmological perturbations on a brane in an anti-de Sitter bulk," hep-th/0012073.

[36] C. van de Bruck, M. Dorca, R. H. Brandenberger, and A. Lukas, Phys. Rev. D 62, 123515 (2000).

[37] K. Koyama and J. Soda, Phys. Rev. D 65, 023519 (2002).

[38] D. Langlois, Phys. Rev. Lett. 86, 2212 (2001).

[39] H. Kodama, A. Ishibashi, and O. Seto, Phys. Rev. D 62, 064022 (2000).

[40] V. Bozza, M. Gasperini, and G. Veneziano, Nucl. Phys. B619, 191 (2001).

[41] D. Langlois, R. Maartens, M. Sasaki, and D. Wands, Phys. Rev. D 63, 084009 (2001).

[42] C. Csaki, M. Graesser, L. Randall, and J. Terning, Phys. Rev. D 62, 045015 (2000).

[43] D. J. Chung, L. L. Everett, and H. Davoudiasl, Phys. Rev. D 64, 065002 (2001).

[44] C. Misner, K. Thorne, and J. Wheeler, Gravitation (Freeman, San Francisco, 1970), p. 965.

[45] C.B. Netterfield et al., Astrophys. J. 571, 604 (2002); R. Stompor et al., Astrophys. J. Lett. 561, L7 (2001); N.W. Halverson et al., Astrophys. J. 568, 38 (2002).

[46] G. D. Starkman, D. Stojkovic, and M. Trodden, Phys. Rev. Lett. 87, 231303 (2001).

[47] J. Khoury, B. A. Ovrut, P. J. Steinhardt, and N. Turok, Phys. Rev. D 64, 123522 (2001).

[48] W. S. Bae, Y. M. Cho, and S. H. Moon, J. High Energy Phys. 03, 039 (2001).

[49] S. H. Moon, S. J. Rey, and Y. B. Kim, Nucl. Phys. B602, 467 (2001).

[50] P. Kraus, J. High Energy Phys. 12, 011 (1999).

[51] Supernova Cosmology Project Collaboration, S. Perlmutter et al., Astrophys. J. 517, 565 (1999).

[52] Supernova Search Team Collaboration, A. G. Riess et al., Astron. J. 116, 1009 (1998). 